\title{
Téoros
}

Revue de recherche en tourisme

\section{Le camping en baisse...}

\section{Richard Cooke et Lucille Lebel}

Volume 6, numéro 1, mars 1987

URI : https://id.erudit.org/iderudit/1080529ar

DOI : https://doi.org/10.7202/1080529ar

Aller au sommaire du numéro

\section{Éditeur(s)}

Université du Québec à Montréal

\section{ISSN}

0712-8657 (imprimé)

1923-2705 (numérique)

Découvrir la revue

Citer cet article

Cooke, R. \& Lebel, L. (1987). Le camping en baisse... Téoros, 6(1), 43-44.

https://doi.org/10.7202/1080529ar d'utilisation que vous pouvez consulter en ligne.

https://apropos.erudit.org/fr/usagers/politique-dutilisation/ 


\title{
Hors-thème
}

\section{Le camping en baisse...}

\author{
par Richard Cooke et Lucille Lebel*
}

Le camping est en baisse au Québec! Les chiffres révèlent une diminution moyenne de $10 \%$ pour l'ensemble des sites gérés par le ministère du Loisir, de la Chasse et de la Pêche à la grandeur du Québec (voir tableau 1). Ce phénomène affecte aussi les terrains de camping privés; en 1980 , Statistique Canada recensait 604 terrains de camping privés répartis sur l'ensemble du territoire québécois, en 1985, il n'en restait plus que 555 .

De plus, il faut préciser que cette baisse de $10 \%$ est une moyenne provinciale, et que ce chiffre peu alarmant à prime abord, cache une réalité, qui pour certaines régions, est beaucoup plus difficile à vivre. Le tableau 3 le démontre très bien, avec des chiffres wfrisants le $30 \%$ en certains endroits.

Assistons-nous à une diminution de l'activité touristique en région périphérique, ou est-ce là le résultat d'un déplacement des préférences du touriste dans le choix de son mode d'hébergement? S'agit-il d'une baisse passagère ou d'une tendance irréversible?

Afin de répondre à cette question, nous avons interrogé les statistiques de fréquentation des bases de plein air et celles des Agricotours. La "parentéw qui existe entre ces différentes clientèles nous permet d'effectuer des comparaisons valables. Dans les deux cas, les chiffres démontrent

TABLEAU 1

Variation du taux d'occupation par région

\begin{tabular}{|l|c|c|c|c|}
\hline \multicolumn{1}{|c|}{ régions anées } & 85 & 84 & 83 & 82 \\
\hline Est du Québec & 30,0 & 30,5 & 35,6 & 44,1 \\
\hline Saquenay & 34,9 & 40,1 & 52,3 & 62,0 \\
\hline Québec & 27,9 & 29,9 & 33,1 & 29,3 \\
\hline Mauricie & 31,8 & 32,3 & 37,7 & 40,7 \\
\hline Estrie & 62,7 & 64,4 & 64,2 & 65,5 \\
\hline Montréal & 25,3 & 31,0 & 31,3 & 38,8 \\
\hline Outaouais & 24,3 & 24,8 & 30,4 & 35,8 \\
\hline Abitibi & 24,2 & 27,6 & 26,1 & 21,7 \\
\hline Cóte-Nord & 23,6 & 19,9 & 27,4 & 34,6 \\
\hline
\end{tabular}

"Richard Cooke travaille au Service du plein air et des parcs du MLCP a la Direction régionale du Saguenay. Lac Saint-Jean. Lucile Lebel est enselgnante dans une polyvalente de Chicoutimi.

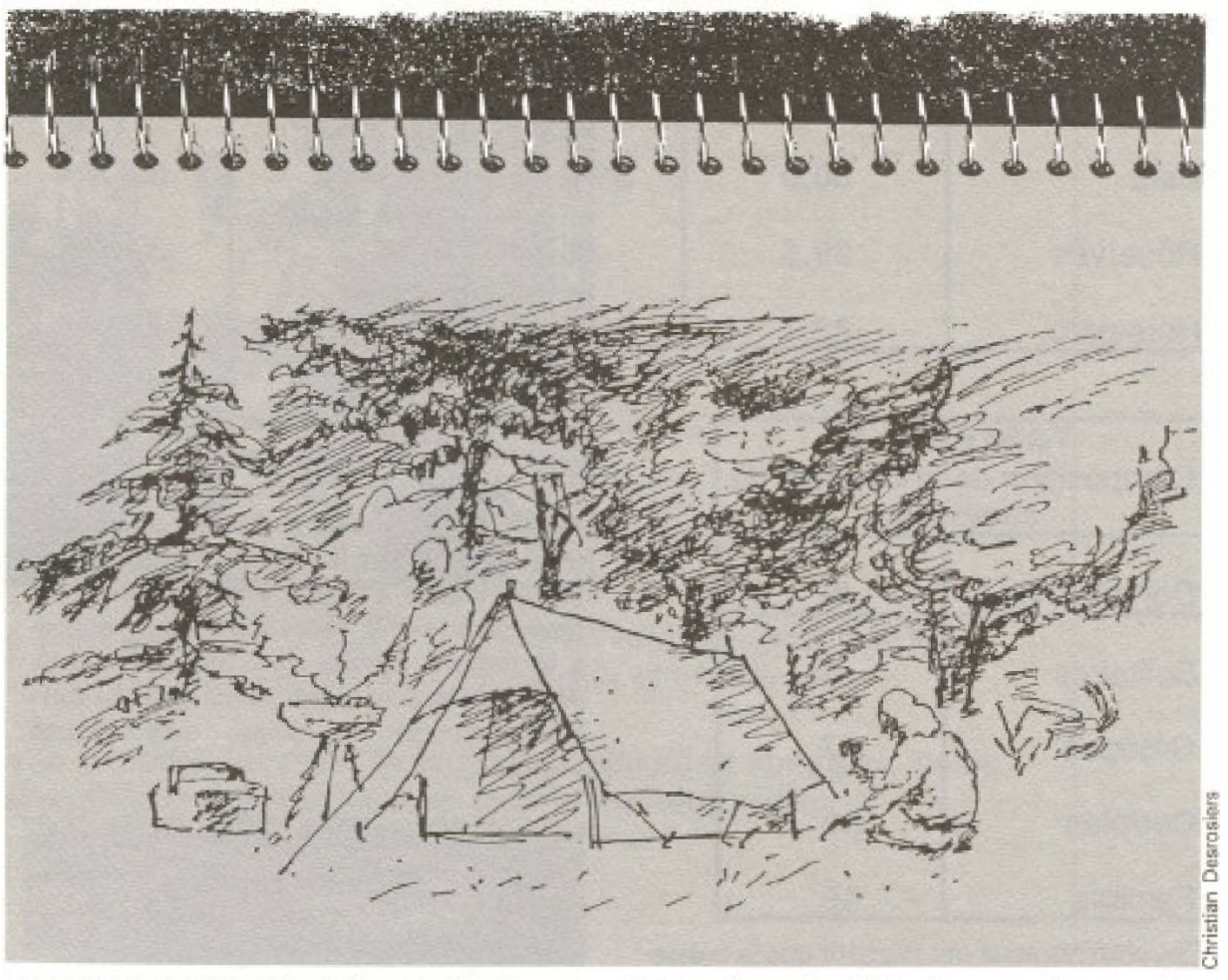

une augmentation des plus significatives: entre les années 1980-1985, les bases de plein air voient leur fréquentation augmenter de $74 \%$. Les gîtes du passant (Agricotours) performent tout aussi bien. Bien que les chiffres ne soient pas encore compilés, développement des Agricotours, nous révélait que la demande des usagers ainsi que les demandes d'accréditation au réseau d'hébergement à la ferme connaissent pour cette mếme période (80-85) une augmentation très forte et soutenue.

Il ne s'agit donc pas d'une baisse de l'activité touristique dans les régions périphériques, mais d'un déplacement des preferences des usagers. Comment expliquer ce déplacement, hasard, mode? Pour tenter de répondre à cette seconde question, nous avons analysé plusieurs variables regroupées en trois catégories: des facteurs Economiques, des facteurs environnementaux et des facteurs d'ordre social. Les deux premières catégories ne nous ont apporté rien de valable. Par contre, au chapitre des facteurs sociaux, la composition de la clientèle de campeurs nous a fourni un filon inté ressant.

Le tableau 4 nous présente les résultats de cette analyse. 
TABLEAU 2

Nombre de terrains de camping privés

opérationnels au Québec

\begin{tabular}{|l|c|c|c|c|c|}
\hline années & 85 & 84 & 83 & 82 & 81 \\
\hline $\begin{array}{l}\text { nombre } \\
\text { de terrains }\end{array}$ & 555 & 556 & 569 & 572 & 604 \\
\hline
\end{tabular}

TABLEAU 3

Variation du taux d"occupation des terrains de camping gérés par le MLCP au Québec

\begin{tabular}{|c|c|c|c|c|}
\hline $\begin{aligned} & \text { lieux } \\
& \text { années } \\
&\end{aligned}$ & 85 & 84 & 83 & 82 \\
\hline Parc & 30,9 & 31,7 & 35,6 & 39,7 \\
\hline Réserves & 29,5 & 28 & 32,3 & 37,7 \\
\hline Hors-parc & 32,7 & 36,6 & 37,8 & 43,6 \\
\hline
\end{tabular}

\begin{tabular}{|l|c|}
\hline \multicolumn{2}{|c|}{$\begin{array}{c}\text { TABLEAU } \\
\text { Composition de la clientèle de } \\
\text { campeurs }\end{array}$} \\
\hline Composition & $\%$ \\
\hline Solitaires & 4 \\
Groupes & 17 \\
Couples & 33 \\
Familles & 46 \\
\hline
\end{tabular}

Ces chiffres nous ont suggéré que la baisse observéc au niveau de la fréquentation vient probablement de l'abandon du camping par une forte proportion de couples et de familles comme mode d'hébergement vacancier. Les $4 \%$ de solitaires et les $17 \%$ de groupes ne pouvant cautionner a eux seuls le volume de la baisse enregistrée. Alors, pourquoi un tel changement est-il irréversible?

La question reste vaste, quoique le sujet d'investigation se précise. Encore une fois, plusieurs variables ont été scrutées; la question des équipements connexes, la proximité et la qualité des plans d'eau, la taille des voitures familiales, le vieillissement de la population, etc.

Nous étions dans un cul-de-sac jusqu'à ce qu'une observation des habitudes des campeurs et surtout une identification du rôle dévolu à chacun des membres de la famille ou du couple nous permettent d'élaborer une hypothese.

Il en est ressorti que la plupart de ces gens transportent en vacances leurs habitudes de vie au foyer. Ceci implique que, à quelques exceptions près, le partage des rôles (travaux domestiques, menage, etc.) reste le metme. Or, le mouvement social actuel soutenu ou forcé par la montée du féministne (hé oui), tend à rejeter cette pratique. Le féminisme, par la liberation qu'il a permise à la femme, semble être une des causes déterminantes du déplacement observé dans l'hebergement touristique actuellement pratiqué.

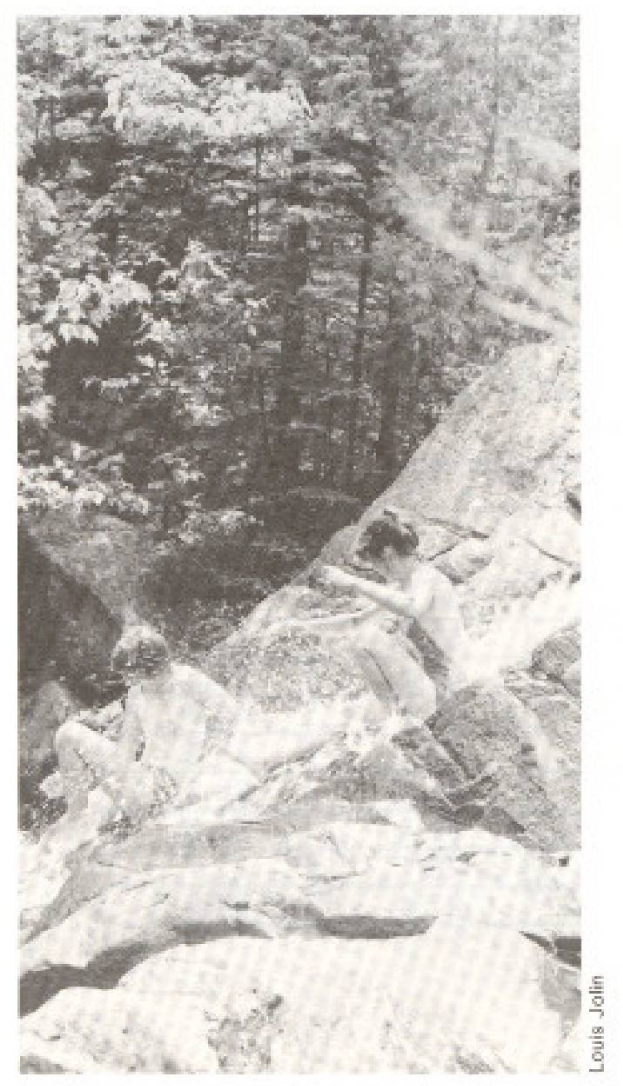

Cette hypothese, qui nous a d'abord fait sourite, s'est rapidement confirmée par le raisonnement suivant: les femmes, maintenant salariées, sont en mesure d'exiger un nouveau partage des rôles. Elles refusent d'être consignées aux corvées ménagères. Or, le camping, par sa nature, impose plusieurs de ces tâches: préparation des repas, entretien de la tente ou de la roulotte, achat d'épicerie... Donc, si le revenu de l'épouse ou de la wchums lui permet d'échapper à la consigne, il lui permet aussi de contribuer au financement d'un type de vacances qui, bien que plus dispendieux (bases de plein air, agricotours), élimine ces thiches dont tous veulent se libérer.

Notre but initial n'était pas de démontrer hors de tout doute raisonnable que la montée du féminisme soit responsable de la baisse de fréquentation des terrains de camping. Nous désirions d'abord et avant tout souligner une tendance au niveau de la consommation d'un important créneau tourtistique. Nous croyons avoir identifié un phénomène que développeurs et promoteurs devraient prendre en compte dans leurs perspectives d'investissement. De plus, nous ayons tente, avec succes espérons-le, de baliser une avenue de recherche dont les résultats seraient sutrement appréciés des propriétaires et des gestionnaires de terrains de camping, confrontés à une baisse de leur marché $f$

\begin{abstract}
Bibliographie
OUELLETTE, Françoise, Romaine Ouellutte, Les groupes de femmes du Québec en 1985: champs d'intervantion, structure et movens d'action. Conseil du statut de la femme, Qudbec, 1966, 314 pages.

MLCP. La fréquentation des territoires ot dquipements du MLCP. Queber, 1985 .

MLCP. Les bases de plein air printemps; Até, automne 1986. Québec 1986.
\end{abstract}

MLCP. Étude sur las prátérenees des campeurs du Qubbec. Quebec 1976.

MLCP, Agricotours - Gites du passant ot formos de vacances. Québec, 1986 .

ELKIN, F. La famille au Canมda. Donnees, recherches et lacunes sur la famile au Canada, Congres canadien de la famillo, Ottawa 1964.

Entravue: Mme Francine Mawnce. Service de développement de la Fédération des Agricotours du Québec. Octobre 1986.

MLCP, Sondage aupros des usagers du camping de Vall Jabert. Jonquibre, 1979 . 